\title{
Association of Night Eating with Depression and Depressive Symptoms in Korean Women
}

\author{
Kyung Won Lee ${ }^{1} \mathbb{D}$ and Dayeon Shin ${ }^{2, *}$ (ID \\ 1 Department of Food Science and Nutrition, Gwangju University, Gwangju 61743, Korea; \\ kwlee@gwangju.ac.kr \\ 2 Department of Food and Nutrition, Inha University, Incheon 22212, Korea \\ * Correspondence: dyshin@inha.ac.kr
}

Received: 29 October 2019; Accepted: 29 November 2019; Published: 1 December 2019 updates

\begin{abstract}
This study examined the associations of night eating with depression and depressive symptoms in Korean adults. The study used a nationally representative sample of 31,690 Korean adults ( $\geq 19$ years old) from the Korea National Health and Nutrition Examination Survey from 2008 to 2013. The participants were divided into two groups based on status of night eating: night eaters (consuming $\geq 25 \%$ of total daily energy intake between 21:00 and 06:00) and non-night eaters. Depression was defined based on diagnosis by a doctor, whereas depressive symptoms were defined as feelings of sadness or desperation for more than two weeks in the last one year. Multivariable logistic regression analyses were performed to examine the relationship between night eating and odds of depression and depressive symptoms after controlling for age, education, income, marital status, drinking, smoking, day of recalled intake, physical activity, body mass index, menopausal status (women only), total energy intake, and sleep duration. A total of $14.3 \%$ of Korean adults were night eaters. Night eaters were more likely to be men, young, less educated, single, drinkers, current smokers, and not employed (all $p \mathrm{~s}<0.05$ ). In women, night eaters had higher odds of depression (adjusted odds ratio [AOR], 1.33; 95\% confidence interval [CI], 1.02-1.75; $p$ for trend $=0.0389$ ) and depressive symptoms (AOR, 1.20; $95 \% \mathrm{CI}, 1.01-1.41 ; p$ for trend $=0.0382$ ) compared with non-night eaters. However, no associations of night eating with depression and depressive symptoms were found in men. Night eaters had higher odds of depression and depressive symptoms only in Korean women. Future studies are warranted to elucidate the underlying psychological and behavioral mechanisms that in turn may shed light on the factors influencing both night eating and odds of depression and depressive symptoms.
\end{abstract}

Keywords: night eating; depression; depressive symptoms; nocturnal ingestion of food; Korea National Health and Nutrition Examination Survey

\section{Introduction}

Depression is one of the most common mental health disorders, affecting more than 300 million people, or $4.4 \%$ of the global population [1]. According to the World Health Organization, depression ranks as the third cause of disease burden worldwide; it is projected to contribute most to the global burden of disease in 2030 [2]. In Korea, depressive disorders have emerged as the fourth leading cause of disability [3]. Given the growing socioeconomic burden of depression, many efforts have been made to identify the factors that can prevent or alleviate depression and depressive symptoms.

As a part of various efforts, researchers have sought to identify dietary factors related to depression. Night eating has been described through various expressions, such as night eating and nocturnal ingestion of food, characterized by a circadian delay in daily food ingestion [4-6]. Since the term "night eating syndrome (NES)" was first introduced by Stunkard et al. in 1955 [7], continuous attention 
has been given to the relationship between night eating and health outcomes. Depressive mood has been found to be more severe among individuals with NES, and prevalence of night eating increases in those with binge eating disorders [8], as well as schizophrenia [9]. A cohort study conducted by Colles et al. [10] found positive associations between NES, body mass index, and binge eating. They also reported that individuals having nocturnal snacking tend to have higher psychological distress [10].

Although studies have examined the relationship between night eating and psychiatric disorders, most of them have focused on night eating as one of the dietary behaviors in patients with psychological disturbance. For this reason, findings from previous studies are limited to patients with psychiatric disorders, and additional investigations focusing on the general population are needed.

Particularly, Korean society has distinct lifestyles, such as frequent overtime work and social gathering, as well as a late-night snacking culture. These lifestyles cause Koreans to consume food later than is usually thought to be dinner time. Given that depression is prevalent and the possibility of exposure to night eating is increased, studies exploring the relationship between night eating and psychiatric disorders among general Korean adult populations are needed. Therefore, we aimed to identify the various factors related to night eating habits and investigate the potential associations of night eating with the odds of depression and depressive symptoms using data from representative samples of the Korean adult population.

\section{Materials and Methods}

\subsection{Data Source and Study Population}

The Korea National Health and Nutrition Examination Survey (KNHANES) is an ongoing national surveillance system that was established to monitor the health, nutritional status, and prevalence of chronic diseases in the Korean population [11,12]. KNHANE uses a multi-stage clustered sampling design to obtain nationally representative samples of non-institutionalized Korean citizens, and consist of three surveys: health interview, health examination including physical examinations and clinical tests, and nutrition survey [13]. A total of 36,081 participants aged 19 years old and older completed the nutrition survey in KNHANES 2008-2013. For the present study, we excluded those who were reporting implausible dietary intake ( $<500$ or $>5000 \mathrm{kcal} /$ day) $(n=570)$, pregnant and lactating women $(n=511)$, not eligible for data analyses because of missing responses on survey questionnaire items related to depression and depressive symptoms, and those with missing information on potential covariates $(\mathrm{n}=3310)$. The final analytical sample of this cross-sectional study included 31,690 Korean adults (12,720 men and 18,970 women).

Informed consent was obtained from all participants, and all of the survey protocols and procedures were approved by the Korea Centers for Disease Control and Prevention (KCDC) Institutional Review Board [11,12].

\subsection{Determination of Night Eating}

We used the 24-h dietary recall data obtained from KNHANES to determine night eating status. During the 24-h dietary recall survey, participants were asked to report a clock time for each reported food or beverage item. The participants were classified into two groups based on the definition of night eating used in previous Korean studies [14-16]: night eaters (consuming $\geq 25 \%$ of total daily energy intake between 21:00 and 06:00) vs. non-night eaters. As a 24-h dietary recall that covered a 24-h time period (00:00 to 23:59) used in the KNHANES was adopted in this study, we defined "night (time)" as the combination of two time periods, 00:00 to 06:00 and 21:00 to 23:59, on the same calendar day.

\subsection{Outcome Variables}

Depressive symptoms were assessed by the following question: "In the last year, have you felt sadness or desperation for more than two weeks?". Participants who answered "yes" were considered 
to have depressive symptoms. In addition, participants were asked if they had been diagnosed with depression by a doctor in their lifetime. Participants who answered "yes" were regarded as having depression.

\subsection{Statistical Analyses}

To account for the complex survey design, all analyses were weighted using the SURVEY procedure with sample weights, strata, and primary sampling units recommended by the KNHANES analytic guidelines [11]. All statistical analyses were performed using SAS (version 9.4; SAS Institute, Inc., Cary, NC, USA). Statistical significance was declared at $p<0.05$.

The general and health-related characteristics, eating behavior, and nutrient intakes of participants were compared across the night eating status using the chi-squared test for categorical variables and multiple linear regressions for continuous variables. This study also used multiple logistic regression analyses to estimate the adjusted odds ratios (ORs) with 95\% confidence intervals (CIs) after adjusting for potential covariates in three models. Model 1 was adjusted for age (years). Model 2 was additionally adjusted for education level (middle school graduates or less, high school graduates, and college graduation or higher), income level (lowest, lowest middle, upper middle, and highest), marital status (married or single), drinking (never/rarely, $\leq 1$ times/month, $>1$ times/month), smoking (non-smokers, former smoker, current smoker), occupation (employed or unemployed), day of recalled intake (Mondays-Thursdays or Fridays-Saturdays), regular physical activity (yes or no), body mass index $\left(\mathrm{kg} / \mathrm{m}^{2}\right)$, and menopausal status (yes or no; women only). Model 3 was additionally adjusted for total energy intake (kcal/day) and sleep duration (hours).

\section{Results}

The general characteristics of the participants according to night eating habits are presented in Table 1. Among the 31,690 participants, 3504 (14.3\%) were night eaters. Relative to non-night eaters, night eaters were more likely to be men, single, and current smokers, but less likely to be employed (all ps $<0.01)$. Night eaters also tended to consume alcohol more than one time per month $(p<0.05)$ and have shorter sleep duration $(p<0.01)$ compared with non-night eaters. However, income, day of recalled intake, and physical activity were not associated with night eating habits.

Table 1. General characteristics of study participants by night eating habits in Korean adults ${ }^{1}$.

\begin{tabular}{|c|c|c|c|c|c|}
\hline & Total & Non-Night Eaters & Night Eaters & $p^{2}$ & Night Eating \\
\hline & $n\left(W^{\prime} d \%\right)$ & n $\left(W^{\prime} t^{\prime} \%\right)$ & n (Wt'd \%) & & AOR $(95 \% \text { CI })^{3}$ \\
\hline Total & $31,690(100.00)^{4}$ & $28,186(85.67)$ & $3504(14.33)$ & & - \\
\hline Sex & & & & $<0.0001 * *$ & \\
\hline Men & $12,720(49.44)$ & $10,890(47.20)$ & $1830(62.87)$ & & 1.00 \\
\hline Women & $18,970(50.56)$ & $17,296(52.80)$ & $1674(37.13)$ & & $1.23(1.08-1.41)^{* *}$ \\
\hline Age (years) & $45.20 \pm 0.17^{5}$ & $46.32 \pm 0.18$ & $38.51 \pm 0.29$ & $<0.0001^{* *}$ & $0.97(0.96-0.97)^{* *}$ \\
\hline Education level & & & & $<0.0001 * *$ & \\
\hline$\leq$ Middle school & $12,331(28.64)$ & $11,459(30.36)$ & $872(18.36)$ & & $1.39(1.19-1.63)^{* *}$ \\
\hline High school & $10,633(39.46)$ & $9150(37.98)$ & $1483(48.29)$ & & $1.26(1.14-1.40)^{* *}$ \\
\hline$\geq$ College & $8726(31.91)$ & 7577 (31.67) & 1149 (33.35) & & 1.00 \\
\hline Income & & & & 0.0780 & \\
\hline Lowest & $7704(25.27)$ & $6877(25.37)$ & 827 (24.68) & & 1.00 \\
\hline Lower middle & $8062(25.65)$ & $7142(25.45)$ & $920(26.82)$ & & $1.12(0.98-1.28)$ \\
\hline Upper middle & $7987(24.84)$ & 7057 (24.63) & $930(26.13)$ & & $1.14(0.99-1.31)$ \\
\hline Highest & $7937(24.24)$ & $7110(24.55)$ & $827(22.38)$ & & $1.01(0.88-1.17)$ \\
\hline
\end{tabular}


Table 1. Cont.

\begin{tabular}{|c|c|c|c|c|c|}
\hline & \multirow{2}{*}{$\begin{array}{c}\text { Total } \\
\text { n }\left(W^{\prime} \mathbf{d}^{\%} \%\right)\end{array}$} & \multirow{2}{*}{$\begin{array}{c}\text { Non-Night Eaters } \\
\mathrm{n}\left(\mathrm{Wt}^{\prime} \mathrm{d} \%\right)\end{array}$} & \multirow{2}{*}{$\begin{array}{c}\text { Night Eaters } \\
n\left(W^{\prime} \mathbf{d} \%\right)\end{array}$} & \multirow{2}{*}{$p^{2}$} & \multirow{2}{*}{$\begin{array}{c}\text { Night Eating } \\
\text { AOR }(95 \% \text { CI })^{3}\end{array}$} \\
\hline & & & & & \\
\hline Marital status & & & & $<0.0001^{* *}$ & \\
\hline Married & $27,679(78.80)$ & 25,051 (81.05) & $2628(65.35)$ & & 1.00 \\
\hline Single & $4011(21.20)$ & $3135(18.95)$ & $876(34.65)$ & & $1.17(1.02-1.34)$ * \\
\hline \multicolumn{4}{|l|}{ Drinking status } & $<0.0001^{* *}$ & \\
\hline Never/rarely & $15,534(42.09)$ & $14,345(44.36)$ & $1189(28.54)$ & & 1.00 \\
\hline$\leq 1$ times/month & $9833(35.05)$ & $8607(34.60)$ & $1226(37.76)$ & & $1.08(0.97-1.21)$ \\
\hline$>1$ times/month & $6323(22.86)$ & $5234(21.04)$ & 1089 (33.69) & & $1.62(1.43-1.85)^{* *}$ \\
\hline \multicolumn{4}{|l|}{ Smoking status } & $<0.0001^{* *}$ & \\
\hline Never & $19,384(54.46)$ & $17,685(56.74)$ & $1699(40.83)$ & & 1.00 \\
\hline Former smoker & $3978(13.60)$ & $3557(13.75)$ & $421(12.67)$ & & $1.14(0.96-1.35)$ \\
\hline Current smoker & $8328(31.95)$ & $6944(29.51)$ & $1384(46.50)$ & & $1.50(1.31-1.72)^{* *}$ \\
\hline \multicolumn{4}{|l|}{ Occupation } & $<0.0001^{* *}$ & \\
\hline Employed & $13,391(37.10)$ & $12,450(38.95)$ & $941(26.04)$ & & $1.48(1.33-1.65)^{* *}$ \\
\hline Not employed & $18,222(62.90)$ & $15,672(61.05)$ & $2550(73.96)$ & & 1.00 \\
\hline \multicolumn{3}{|l|}{$\begin{array}{l}\text { Day of week of dietary } \\
\text { intake }\end{array}$} & & 0.1422 & \\
\hline Monday-Thursday & $18,983(59.66)$ & $16,948(59.92)$ & $2035(58.11)$ & & 1.00 \\
\hline Friday-Saturday & $12,707(40.34)$ & $11,238(40.08)$ & $1469(41.89)$ & & $1.06(0.96-1.17)$ \\
\hline \multicolumn{4}{|l|}{$\begin{array}{l}\text { Regular physical } \\
\text { activity } 6\end{array}$} & 0.0514 & \\
\hline Yes & $15,887(50.84)$ & $14,081(50.54)$ & $1806(52.68)$ & & $0.95(0.87-1.04)$ \\
\hline No & $15,801(49.16)$ & $14,104(49.46)$ & $1697(47.32)$ & & 1.00 \\
\hline $\begin{array}{l}\text { Body mass index } \\
\left(\mathrm{kg} / \mathrm{m}^{2}\right)\end{array}$ & $23.66 \pm 0.03$ & $23.69 \pm 0.03$ & $23.50 \pm 0.08$ & $0.0150 *$ & $0.99(0.97-0.99)$ * \\
\hline \multicolumn{4}{|l|}{ Sleep duration (h) } & 0.4249 & \\
\hline$\leq 6$ (short) & $1311(40.30)$ & $11,664(40.26)$ & $1469(40.54)$ & & $1.14(1.04-1.25) * *$ \\
\hline $6<$ to $<9$ (adequate) & $16,064(52.11)$ & $14,305(52.26)$ & $1759(51.24)$ & & 1.00 \\
\hline$\geq 9$ (long) & $2493(7.59)$ & $2217(7.48)$ & $276(8.22)$ & & $1.13(0.95-1.35)$ \\
\hline \multicolumn{6}{|c|}{$\begin{array}{l}{ }^{1} \text { Data were from the Korea National Health and Nutrition Examination Surveys (KNHANES). All data were } \\
\text { weighted to account for the complex study design according to the analytical guidelines of the KNHANES. }{ }^{*} p<0.05 \text {, } \\
* * p<0.01{ }^{3} \text { AOR, adjusted odds ratio; } 95 \% \text { CI, } 95 \% \text { confidence interval. Multiple logistic regression analysis was } \\
\text { performed to estimate the odds for night eating for the study participants from the KNHANES 2008-2013: statistical } \\
\text { model was adjusted for sex (men and women), age (continuous), education level (middle school graduates or } \\
\text { less, high school graduates, and college graduation or higher), income (lowest, lowest middle, upper middle, and } \\
\text { highest), marital status (married or single), drinking status (never/rarely, } \leq 1 \text { times/month, and }>1 \text { times/month), } \\
\text { smoking status (non-smokers, former smoker, or current smoker), occupation (employed or not employed), day of } \\
\text { week of dietary intake (Monday-Thursday and Friday-Saturday), regular physical activity (yes or no), body mass } \\
\text { index (continuous, kg/m }{ }^{2} \text { ), menopausal status (yes or no, women only), and sleep duration ( } \leq 6,6<\text { to }<9, \text { or } \geq 9 \text { h). } \\
4 \text { Values represented frequency (Wt'd } \% \text { ). }{ }^{5} \text { Mean } \pm \mathrm{SE}^{6} \text { Having regular physical activity was defined as walking } \\
\geq 5 \text { time a week for } \geq 30 \text { min each time. }\end{array}$} \\
\hline
\end{tabular}

Eating behaviors according to night eating habits are presented in Table 2. Night eating habits were negatively associated with the main meal episodes but positively associated with snack episodes and length of eating period in both men and women. The number of main meal episodes and energy intake from main meals were significantly lower in night eaters compared with non-night eaters. In the contrast, night eaters reported higher number of total snack episodes and percentage of energy intake from night main meals, snacks, and night snacks (all $p s<0.01$ ). In addition, time difference between the first and last eating episode differed according to night eating habits, with night eaters reporting a significantly longer ingestion period $(p<0.01)$. Compared with non-night eaters, a higher percentage of night eaters reported having breakfast, lunch, and all three main meals. The percentage of individuals having $\geq 25 \%$ of energy from snacks was more than two times higher in night eaters than non-night eaters. 
Table 2. Eating behaviors by night eating habits in Korean adults ${ }^{1}$.

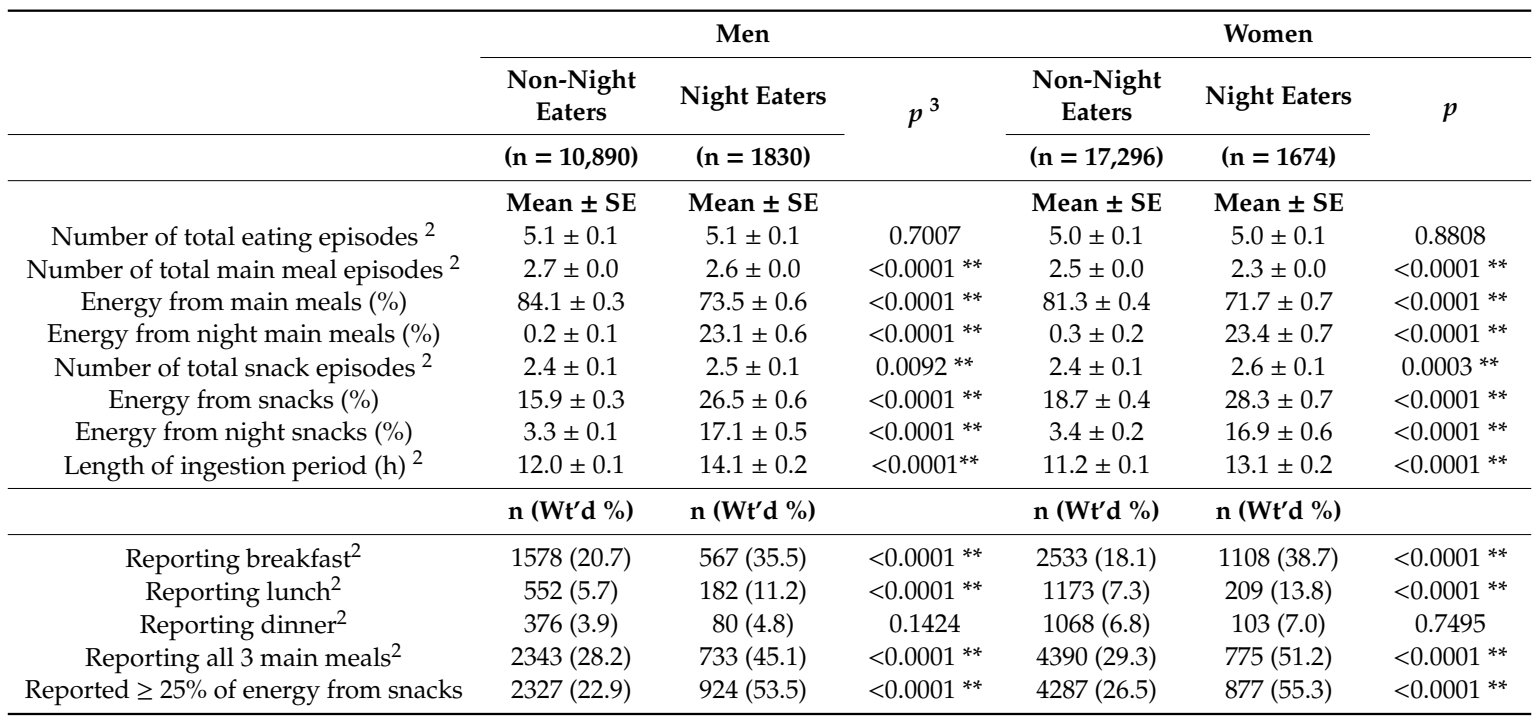

${ }^{1}$ Data were from the Korea National Health and Nutrition Examination Surveys (KNHANES). All data were weighted to account for the complex study design according to the analytical guidelines of the KNHANES. The multivariable linear regression models included covariates including age (continuous), education level (middle school graduates or less, high school graduates, and college graduation or higher), income (lowest, lowest middle, upper middle, and highest), marital status (married or single), drinking status (never/rarely, $\leq 1$ times/month, and $>1$ times/month), smoking status (non-smokers, former smoker, or current smoker), occupation (employed or not employed), day of week of dietary intake (Monday-Thursday and Friday-Saturday), regular physical activity (yes or no), body mass index (continuous, $\mathrm{kg} / \mathrm{m}^{2}$ ), and menopausal status (yes or no, women only). ${ }^{2}$ The models also included total daily energy (continuous) intake as an independent variable ${ }^{3} p$ values were obtained from the multivariable linear regression analyses indicate the significance of the association of each independent variable with night eating habits $(* * p<0.01)$.

Table 3 shows the total energy and nutrient intake by night eating habits. In both sexes, night eaters had a higher energy intake compared with non-night eaters (men: 2262 vs. $2139 \mathrm{kcal}, p<0.01$; women: 1761 vs. $1641 \mathrm{kcal}, p<0.01$ ). Night eating habits were positively associated with the percentage of energy from fat and sodium intake (all $p \mathrm{~s}<0.01$ ). In contrast, night eaters showed lower intakes of carbohydrates, dietary fibers, potassium, calcium, and phosphorus (all $p \mathrm{~s}<0.01$ ).

The multivariable-adjusted ORs for depression and depressive symptoms for night eaters and non-night eaters are listed in Table 4 . In mode 3 , the fully adjusted models, compared with non-night eaters, night eaters showed higher odds for depression (AOR, $1.33 ; 95 \%$ CI, 1.02-1.75; $p$ for trend $=$ 0.0389 ) and depressive symptoms (AOR, $1.20 ; 95 \% \mathrm{CI}, 1.01-1.41 ; p$ for trend $=0.0382$ ) only in women (Table 4). 
Table 3. Total energy and nutrient intake by night eating habits in Korean adults ${ }^{1}$.

\begin{tabular}{|c|c|c|c|c|c|c|c|}
\hline & & \multicolumn{3}{|c|}{ Men } & \multicolumn{3}{|c|}{ Women } \\
\hline & & Non-Night Eaters & Night Eaters & \multirow{2}{*}{$p^{3}$} & Non-Night Eaters & Night Eaters & \multirow{2}{*}{$p$} \\
\hline & 2015 KDRIs $^{4}$ & $(n=10,890)$ & $(n=1830)$ & & $(\mathrm{n}=17,296)$ & $(\mathrm{n}=1674)$ & \\
\hline & & Mean \pm SE & Mean \pm SE & & Mean \pm SE & Mean \pm SE & \\
\hline Total energy, kcal & & $2139 \pm 15$ & $2262 \pm 27$ & $<0.0001^{* *}$ & $1641 \pm 17$ & $1761 \pm 24$ & $<0.0001^{* *}$ \\
\hline Carbohydrate, $\%$ of energy ${ }^{2}$ & $55-65$ & $63.7 \pm 0.2$ & $60.3 \pm 0.3$ & $<0.0001 * *$ & $68.9 \pm 0.1$ & $63.9 \pm 0.4$ & $<0.0001^{* *}$ \\
\hline Protein, $\%$ of energy ${ }^{2}$ & $7-20$ & $14.4 \pm 0.2$ & $14.2 \pm 0.2$ & 0.0860 & $14.1 \pm 0.1$ & $14.2 \pm 0.2$ & 0.6845 \\
\hline Fat, $\%$ of energy 2 & $15-30$ & $17.9 \pm 0.1$ & $18.9 \pm 0.2$ & $<0.0001^{* *}$ & $17.2 \pm 0.1$ & $19.5 \pm 0.3$ & $<0.0001^{* *}$ \\
\hline Dietary fiber, $\mathrm{g}^{2}$ & M: 25, W: 21 & $8.1 \pm 0.2$ & $7.3 \pm 0.2$ & $<0.0001^{* *}$ & $7.0 \pm 0.1$ & $6.1 \pm 0.1$ & $<0.0001^{* *}$ \\
\hline Sodium, $\mathrm{mg}^{2}$ & 1100-1500 & $5249 \pm 71$ & $5612 \pm 33.5$ & $<0.0001^{* *}$ & $3761 \pm 63$ & $4040 \pm 23$ & $<0.0001^{* *}$ \\
\hline Potassium, $\mathrm{mg}^{2}$ & 3500 & $553.6 \pm 3.6$ & $517.4 \pm 7.8$ & $<0.0001^{* *}$ & $453.9 \pm 2.7$ & $417.1 \pm 7.0$ & $<0.0001^{* *}$ \\
\hline Calcium, $\mathrm{mg}^{2}$ & $700-800$ & $3337 \pm 14$ & $3100 \pm 31$ & $<0.0001^{* *}$ & $1002 \pm 2.6$ & $944 \pm 8$ & $<0.0001^{* *}$ \\
\hline Phosphorus, $\mathrm{mg}^{2}$ & 700 & $1310 \pm 4.0$ & $1241 \pm 9.2$ & $<0.0001^{* *}$ & $2736 \pm 12.8$ & $2495 \pm 32$ & $<0.0001^{* *}$ \\
\hline
\end{tabular}

${ }^{1}$ Data were from the Korea National Health and Nutrition Examination Surveys (KNHANES). All data were weighted to account for the complex study design according to the analytical guidelines of the KNHANES. The multivariable linear regression models included covariates including age (continuous), education level (middle school graduates or less, high school graduates, and college graduation or higher), income (lowest, lowest middle, upper middle, and highest), marital status (married or single), drinking status (never/rarely, $\leq 1$ times/month, and $>1$ times/month), smoking status (non-smokers, former smoker, or current smoker), occupation (employed or not employed), day of week of dietary intake (Monday-Thursday and Friday-Saturday), regular physical activity (yes or no), body mass index (continuous, $\mathrm{kg} / \mathrm{m}^{2}$ ), and menopausal status (yes or no, women only). ${ }^{2}$ The models also included total daily energy (continuous) intake as an independent variable ${ }^{3} p$ values were obtained from the multivariable linear regression analyses indicate the significance of the association of each independent variable with night eating habits $\left({ }^{* *} p<0.01\right) .{ }^{4}$ KDRIs, Dietary Reference Intakes for Koreans. 
Table 4. The adjusted odds ratios (AORs) and 95\% confidence intervals (CIs) for depression and depressive symptoms by night eating habits in Korean adults ${ }^{1}$.

\begin{tabular}{|c|c|c|c|c|c|c|c|}
\hline & & \multicolumn{3}{|c|}{ Men } & \multicolumn{3}{|c|}{ Women } \\
\hline & & Non-night Eaters & Night Eaters & \multirow{2}{*}{$p^{3}$} & Non-Night Eaters & Night Eaters & \multirow{2}{*}{$p$} \\
\hline & & $(\mathrm{n}=10,890)$ & $(n=1830)$ & & $(n=17,296)$ & $(n=1674)$ & \\
\hline \multirow{4}{*}{ Depression $^{2}$} & & & AOR $(95 \%$ CI) & & & AOR $(95 \%$ CI) & \\
\hline & Model 1 & 1.00 & $0.82(0.48-1.40)$ & 0.4609 & 1.00 & $1.44(1.10-1.88)$ & $0.0077^{* *}$ \\
\hline & Model 2 & 1.00 & $0.82(0.47-1.41)$ & 0.4669 & 1.00 & $1.34(1.02-1.76)$ & 0.0360 * \\
\hline & Model 3 & 1.00 & $0.81(0.47-1.39)$ & 0.4405 & 1.00 & $1.33(1.02-1.75)$ & $0.0389 *$ \\
\hline \multirow[t]{3}{*}{$\begin{array}{l}\text { Depressive } \\
\text { symptoms }\end{array}$} & Model 1 & 1.00 & $1.06(0.87-1.30)$ & 0.5554 & 1.00 & $1.31(1.11-1.55)$ & $0.0015^{* *}$ \\
\hline & Model 2 & 1.00 & $1.01(0.82-1.24)$ & 0.9371 & 1.00 & $1.19(1.01-1.40)$ & $0.0476^{*}$ \\
\hline & Model 3 & 1.00 & $1.01(0.82-1.24)$ & 0.9241 & 1.00 & $1.20(1.01-1.41)$ & $0.0382 *$ \\
\hline
\end{tabular}

${ }^{1}$ Data were from the Korea National Health and Nutrition Examination Surveys (KNHANES). All data were weighted to account for the complex study design according to the analytical guidelines of the KNHANES. AOR, adjusted odds ratio; $95 \%$ CI, $95 \%$ confidence interval. Multiple logistic regression analysis was performed to estimate the odds ratio for depression and depressive symptoms for the study participants from the KNHANES 2008-2013, in three models: The Model 1 was adjusted for sex (men and women) and age (continuous); Model 2 was additionally adjusted for education level (middle school graduates or less, high school graduates, and college graduation or higher), income (lowest, lowest middle, upper middle, and highest), marital status (married or single), drinking status (never/rarely, $\leq 1$ times/month, and $>1$ times/month), smoking status (non-smokers, former smoker, or current smoker), accupation (employed or not employed), day of week of dietary intake (Monday-Thursday and Friday-Saturday), regular physical activity (yes or no), body mass index (continuous, occupation (employed or not employed), day of week of dietary intake (Monday-Thursday and Friday-Saturday), regular physical activity (yes or no), body mass index (continuous,
$\mathrm{kg} / \mathrm{m}^{2}$ ), and menopausal status (yes or no, women only); Model 3 was additionally adjusted for total energy intake (continuous) and sleep duration (continuous). ${ }^{2}$ Depression was defined based on self-reported doctor-diagnosed depression, and depressive symptoms were defined as feelings of sadness or desperation for $\geq 2$ weeks during the past 1 year. ${ }^{3} p$ values were obtained from the multivariable logistic regression models with diagnosis of depression or depressive symptoms as the outcome variable $\left({ }^{*} p<0.05,{ }^{* *} p<0.01\right)$ 


\section{Discussion}

In this large cross-sectional study of free-living Korean adults, we explored the factors related night eating behaviors and the association of night eating status with depression and depressive symptoms. Our findings showed that $14.3 \%$ of Korean adults consumed more than $25 \%$ of their total daily energy intake between 21:00 and 06:00. Participants who were men, low educated, single, current smokers, not currently employed, and consumed alcohol $\geq 1$ times/month were more likely to have a night eating habit compared with their counterpart (Table 1). This corresponded with the results from a previous study of Korean adolescents who participated in KNHANES [14]. Moreover, night eating habits were positively associated with depression and depressive symptoms. Interestingly, these associations were observed only in women, and remained statistically significant after controlling for potential covariates.

Earlier research has indicated that morning meals usually have carbohydrate-rich features, lunch meals are rich in protein, and evening meals have high fat contents [17]. In other words, evening meals show a high energy density compared with morning meals [18]. In addition, whereas early intake of the day is linked to overall decreases in food intake, intake at late evening increases overall food intake [18]. Similarly, in our study, night eaters reported a higher daily total energy intake and a higher percentage of energy from fat, as well as a lower percentage of energy from carbohydrates. Consistent with our findings, Lundgren et al. [19] reported that night eaters have more daily energy intake (night eaters: $2285 \mathrm{kcal}$ vs. control: $1856 \mathrm{kcal}$ ) and nocturnal food ingestion compared with controls. They also reported that the time point when night eaters consume $75 \%$ of their daily total energy is four hours later compared with controls [19]. The type of foods eaten late at night tends to be unhealthy, palatable foods with high sugar, salt, and fat content, rather than healthy foods, such as fruits and vegetables [20,21]. Likewise, the results of our study indicated that night eaters had lower consumption of dietary fiber, potassium, calcium, and phosphorus, but higher consumption of fats and sodium compared with non-night eaters.

Epidemiological studies have investigated the associations of night eating or NES with mental health. Our results indicating positive relationships between night eating, depression, and depressive symptoms are in agreement with previous studies. A previous cross-sectional study of 404 Korean female nurses also found that NES is significantly associated with the increased odds of depressive symptoms [22]. A US study of overweight individuals reported that night eaters show distinct psychopathological patterns, such as greater depression and lower self-esteem. Another study, in which the two groups had no differences in amount of food intake, reported that night eaters feel less hunger and more full compared with non-night eaters [23]. Another cohort study of 431 Australian aged 18-71 years reported that participants with NES, who reported nocturnal snacking, tend to manifest higher symptoms of depression and hunger along with decreased mental health-related quality of life [10]. In our study, night eaters consumed approximately $20 \%$ of their daily total energy intake from night snacking, which was more than five times compared with non-night eaters. Combined with the previous findings, our results suggest that night eating and snacking might be linked to other psychological disturbances beyond depression and depressive symptoms.

The mechanisms underlying the relationships between night eating and depression and depressive symptoms remain unclear. Their relationships can be explained by three plausible pathways. First, night eating behaviors could contribute to the increased odds of depression or depressive symptoms. Particularly, night eaters are more likely to experience alterations in endogenous nocturnal hormones, namely, melatonin and leptin. According to Birketvedt et al. [4], night eaters have lower plasma melatonin and leptin levels, and higher levels of plasma cortisol relative to non-night eaters. Melatonin is a hormone that plays a role in inducing and maintaining sleep; lowered melatonin levels may lead to impaired sleep and depression [24]. In addition, the slowdown of the nocturnal increases in leptin among night eaters might lead to depression or depressive symptoms. Leptin, which helps suppress appetite and food intake and maintain sleep, tends to increase at night [25-27]. In our study, significant associations between night eating habits and odds of sleep duration were observed in 
women but not in men. In the fully adjusted model (model 3), night eaters were more likely to have a short sleep duration $(\leq 6 \mathrm{~h})(\mathrm{AOR}, 1.20 ; 95 \% \mathrm{CI}, 1.06-1.36$; $p$ for trend $=0.0043$ ) compared with non-night eaters (Supplementary Table S1). Thus, night eating could contribute not only to depressive disorders but to insufficient or excessive sleep duration. Decreased nocturnal leptin levels owing to night eating can interfere with sleep or promote appetite, which in turn leads to a vicious cycle of night eating, impaired sleep, and depressive disorders. Second, night eating could be a means of expressing underlying depression or depressive symptoms. Orhan et al. (2011) demonstrated that prevalence of NES was higher in depressed patients compared to healthy control participants $(35.2 \%$ vs. 19.2\%) [28]. Other two studies from the United States reported that the higher risk for NES was observed among psychiatric clinic outpatients [29] and obese people with serious mental illness [30]. Third, common underlying factors such as insomnia might lead to both night eating behavior and depression or depressive symptoms. Rogers et al. (2006) indicated that insomnia or sleep disturbances precedes NES, and nocturnal eating which is a feature of NES causes sleep disturbances in people with NES [31]. In addition, another study of psychiatric outpatients reported that sleep disturbance was a significant predictor of NES [32]. We also found that individuals with short sleep duration $(\leq 6 \mathrm{~h})$ tended to have night eating compared with those with adequate sleep duration $(6<$ to $<9 \mathrm{~h})$. Due to the nature of cross-sectional design, this study provided cross-sectional association between night eating and depression or depressive symptoms. Additional investigations using prospective cohort study are needed to determine whether night eating causes depression or vice versa.

To our knowledge, this study is the first to identify the associations of night eating status with depression and depressive symptoms in a representative sample of Korean adults. Therefore, results of the current study can be applicable to general Korean adult populations. In addition, we demonstrated that nocturnal ingestion of food could be a risk factor for depression and depressive symptoms in Korean women even after adjusting for various covariates.

The limitations of this study should also be considered. First, as KNHANES did not carry out a survey on NES, we defined night eating according to the time period of food intake or the percentage of energy consumed in a certain time period. Various definitions of night eating have been used in studies conducted in Western countries [33-35]. However, as people usually eat later in the day in Korea compared with the Westerners, this study defined night eating as a later time period than that used in Western studies. To date, a consensus on the operational definition of night eating has not been proposed; additional investigations using a NES questionnaire are warranted for a better understanding of the relationship between NES and depression in Korean populations. Second, KNHANES collected the information on depression and depressive symptoms by self-reports with simple questions. The definitions of depression and depressive symptoms used in the current work have been used widely to identify associations with dietary intakes and behaviors in previous studies of Koreans. However, to yield more accurate results, standardized tools, such as the Patient Health Questionnaire-9 [36], Beck Depression Inventory-II [37], and Center for Epidemiologic Studies Depression Scale [38], should be used in future investigations. Lastly, our cross-sectional analysis could not derive causal relationships of night eating status with depression and depressive symptoms. Further prospective cohort studies are needed to investigate these causal relationships among Korean adults.

\section{Conclusions}

Night eating was significantly associated with increased odds for depression and depressive symptoms in Korean women, but not in men. Several sociodemographic and health-related factors were related to night eating status. The results of this study could help establish dietary behavioral strategies for mental health. Future studies need to elucidate the underlying psychological and behavioral mechanisms that may link the factors influencing night eating and odds of depression and depressive symptoms. 
Supplementary Materials: The following are available online at http://www.mdpi.com/1660-4601/16/23/4831/s1, Table S1: The adjusted odds ratios (AORs) and 95\% confidence intervals (CIs) for short or long sleep duration by night eating habits in Korean adults.

Author Contributions: Conceptualization, K.W.L. and D.S.; methodology, K.W.L.; statistical analysis, K.W.L.; data interpretation, K.W.L. and D.S.; writing—original draft preparation, K.W.L.; writing—review and editing, K.W.L. and D.S.; funding acquisition, K.W.L.

Funding: This study was conducted by research funds from Gwangju University in 2019.

Acknowledgments: The article was prepared using KNHANES 2008-2013 data obtained from the KCDC. All authors appreciate the KCDC for the availability of the KNHANES survey data.

Conflicts of Interest: The authors declare no conflict of interest.

\section{References}

1. World Health Organization. Depression and Other Common Mental Disorders: Global Health Estimates; World Health Organization: Geneva, Switzerland, 2017.

2. Mathers, C. The Global Burden of Disease: 2004 Update; World Health Organization: Geneva, Switzerland, 2008.

3. Institute for Health Metrics and Evaluation. What Health Problems Cause the Most Disability? Available online: http://www.healthdata.org/south-korea (accessed on 10 September 2019).

4. Birketvedt, G.S.; Florholmen, J.; Sundsfjord, J.; Østerud, B.; Dinges, D.; Bilker, W.; Stunkard, A. Behavioral and neuroendocrine characteristics of the night-eating syndrome. Jama 1999, 282, 657-663. [CrossRef]

5. Goel, N.; Stunkard, A.J.; Rogers, N.L.; Van Dongen, H.P.; Allison, K.C.; O’Reardon, J.P.; Ahima, R.S.; Cummings, D.E.; Heo, M.; Dinges, D.F. Circadian rhythm profiles in women with night eating syndrome. J. Biol. Rhythm. 2009, 24, 85-94. [CrossRef]

6. Milano, W.; De Rosa, M.; Milano, L.; Capasso, A. Night eating syndrome: An overview. J. Pharm. Pharmacol. 2012, 64, 2-10. [CrossRef]

7. Stunkard, A.J.; Grace, W.J.; Wolff, H.G. The night-eating syndrome: A pattern of food intake among certain obese patients. Am. J. Med. 1955, 19, 78-86. [CrossRef]

8. Lundgren, J.D.; McCune, A.; Spresser, C.; Harkins, P.; Zolton, L.; Mandal, K. Night eating patterns of individuals with eating disorders: Implications for conceptualizing the night eating syndrome. Psychiatry Res. 2011, 186, 103-108. [CrossRef]

9. Palmese, L.B.; DeGeorge, P.C.; Ratliff, J.C.; Srihari, V.H.; Wexler, B.E.; Krystal, A.D.; Tek, C. Insomnia is frequent in schizophrenia and associated with night eating and obesity. Schizophr. Res. 2011, 133, 238-243. [CrossRef]

10. Colles, S.; Dixon, J.; O’Brien, P.E. Night eating syndrome and nocturnal snacking: Association with obesity, binge eating and psychological distress. Int. J. Obes. 2007, 31, 1722-1730. [CrossRef] [PubMed]

11. Korea Centers for Disease Control and Prevention. User Guide for the Fourth Korea National Health and Nutrition Examination Survey (KNHANES V); Korea Centers for Disease Control and Prevention: Cheongwon, Korea, 2016.

12. Ministry of Health and Welfare, Korea Centers for Disease Control and Prevention. Korea Health Statistics 2012: Korea National Health and Nutrition Examination Survey (KNHANES V-3); Korea Centers for Disease Control and Prevention: Cheongwon, Korea, 2013.

13. Kweon, S.; Kim, Y.; Jang, M.-J.; Kim, Y.; Kim, K.; Choi, S.; Chun, C.; Khang, Y.-H.; Oh, K. Data resource profile: The Korea national health and nutrition examination survey (KNHANES). Int. J. Epidemiol. 2014, 43, 69-77. [CrossRef] [PubMed]

14. Hernandez, E.; Kim, M.; Kim, W.G.; Yoon, J. Nutritional aspects of night eating and its association with weight status among Korean adolescents. Nutr. Res. Pract. 2016, 10, 448-455. [CrossRef] [PubMed]

15. Suh, Y.; Lee, E.-K.; Chung, Y.-J. Comparison of nutritional status by energy level of night snack in Korean adults: Using the data from 2005 Korean National Health and Nutrition Examination Survey. Korean J. Nutr. 2012, 45, 479-488. [CrossRef]

16. Jung, H.J.; Park, K.H.; Cho, Y.G.; Cho, J.J.; Paek, Y.J.; Song, H.J.; Byun, J.S. Night eating tendency and related health risk factors. Korean J. Obes. 2006, 15, 98. 
17. Westerterp-Plantenga, M.; Ijedema, M.; Wijckmans-Duijsens, N. The role of macronutrient selection in determining patterns of food intake in obese and non-obese women. Eur. J. Clin. Nutr. 1996, 50, 580-591. [PubMed]

18. De Castro, J.M. The time of day of food intake influences overall intake in humans. J. Nutr. 2004, 134, $104-111$. [CrossRef] [PubMed]

19. Lundgren, J.D.; Allison, K.C.; O’Reardon, J.P.; Stunkard, A.J. A descriptive study of non-obese persons with night eating syndrome and a weight-matched comparison group. Eat. Behav. 2008, 9, 343-351. [CrossRef]

20. Baron, K.G.; Reid, K.J.; Kern, A.S.; Zee, P.C. Role of sleep timing in caloric intake and BMI. Obesity 2011, 19, 1374-1381. [CrossRef]

21. Gallant, A.; Lundgren, J.; Drapeau, V. Nutritional aspects of late eating and night eating. Curr. Obes. Rep. 2014, 3, 101-107. [CrossRef]

22. Kim, O.-S.; Kim, M.; Lee, J.; Jung, H. Night-eating syndrome and the severity of self-reported depressive symptoms from the Korea Nurses' Health Study: Analysis of propensity score matching and ordinal regression. Public Health 2016, 141, 80-87. [CrossRef]

23. Gluck, M.E.; Geliebter, A.; Satov, T. Night eating syndrome is associated with depression, low self-esteem, reduced daytime hunger, and less weight loss in obese outpatients. Obes. Res. 2001, 9, 264-267. [CrossRef]

24. Rahman, S.A.; Marcu, S.; Kayumov, L.; Shapiro, C.M. Altered sleep architecture and higher incidence of subsyndromal depression in low endogenous melatonin secretors. Eur. Arch. Psychiatry Clin. Neurosci. 2010, 260, 327-335. [CrossRef]

25. Antonijevic, I.; Murck, H.; Frieboes, R.-M.; Horn, R.; Brabant, G.; Steiger, A. Elevated nocturnal profiles of serum leptin in patients with depression. J. Psychiatr. Res. 1998, 32, 403-410. [CrossRef]

26. Atmaca, M.; Kuloglu, M.; Tezcan, E.; Ustundag, B.; Bayik, Y. Serum leptin and cholesterol levels in patients with bipolar disorder. Neuropsychobiology 2002, 46, 176-179. [CrossRef] [PubMed]

27. Lu, X.-Y. The leptin hypothesis of depression: A potential link between mood disorders and obesity? Curr. Opin. Pharmacol. 2007, 7, 648-652. [CrossRef] [PubMed]

28. Orhan, F.O.; Ozer, U.G.; Ozer, A.; Altunoren, O.; Çelik, M.; Karaaslan, M.F. Night eating syndrome among patients with depression. Isr. J. Psychiatry Relat. Sci. 2011, 48, 212-217. [PubMed]

29. Lundgren, J.D.; Allison, K.C.; Crow, S.; O’Reardon, J.P.; Berg, K.C.; Galbraith, J.; Martino, N.S.; Stunkard, A.J. Prevalence of the night eating syndrome in a psychiatric population. Am. J. Psychiatry 2006, 163, 156-158. [CrossRef]

30. Lundgren, J.D.; Rempfer, M.V.; Brown, C.E.; Goetz, J.; Hamera, E. The prevalence of night eating syndrome and binge eating disorder among overweight and obese individuals with serious mental illness. Psychiatry Res. 2010, 175, 233-236. [CrossRef]

31. Kucukgoncu, S.; Tek, C.; Bestepe, E.; Musket, C.; Guloksuz, S. Clinical features of night eating syndrome among depressed patients. Eur. Eat. Disord. Rev. 2014, 22, 102-108. [CrossRef]

32. Rogers, N.L.; Dinges, D.F.; Allison, K.C.; Maislin, G.; Martino, N.; O’Reardon, J.P.; Stunkard, A.J. Assessment of sleep in women with night eating syndrome. Sleep 2006, 29, 814-819. [CrossRef]

33. Striegel-Moore, R.H.; Thompson, D.; Franko, D.L.; Barton, B.; Affenito, S.; Schreiber, G.B.; Daniels, S.R. Definitions of night eating in adolescent girls. Obes. Res. 2004, 12, 1311-1321. [CrossRef]

34. Adami, G.F.; Meneghelli, A.; Scopinaro, N. Night eating and binge eating disorder in obese patients. Int. J. Eat. Disord. 1999, 25, 335-338. [CrossRef]

35. Striegel-Moore, R.H.; Franko, D.L.; Thompson, D.; Affenito, S.; Kraemer, H.C. Night eating: Prevalence and demographic correlates. Obesity 2006, 14, 139-147. [CrossRef]

36. Löwe, B.; Unützer, J.; Callahan, C.M.; Perkins, A.J.; Kroenke, K. Monitoring depression treatment outcomes with the patient health questionnaire-9. Med. Care 2004, 42, 1194-1201. [PubMed]

37. Beck, A.T.; Steer, R.A.; Brown, G.K. Beck depression inventory-II. San Antonio 1996, 78, 490-498.

38. Eaton, W.W.; Muntaner, C.; Smith, C.; Tien, A.; Ybarra, M. Center for Epidemiologic Studies Depression Scale: Review and Revision (CESD and CESD-R). In The Use of Psychological Testing for Treatment Planning and Outcomes Assessment, 3rd ed.; Maruish, M.E., Ed.; Lawrence Erlbaum: Mahwah, NJ, USA, 2004; pp. 363-377.

(C) 2019 by the authors. Licensee MDPI, Basel, Switzerland. This article is an open access article distributed under the terms and conditions of the Creative Commons Attribution (CC BY) license (http://creativecommons.org/licenses/by/4.0/). 